Recepción: 16 / 04 / 2018

Aceptación: 12 / 05 / 2018

Publicación: 02 / 07 / 2018

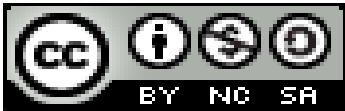

Ciencias económicas y empresariales Artículo de Investigación

\title{
Los ingresos fiscales en la emisión de carbono, biodiversidad y la degradación evitada, como alternativa a la arquitectura financiera
}

\section{Tax revenues in carbon emissions, biodiversity and avoided degradation, as an alternative to the financial architecture}

\section{Receitas fiscais em emissões de carbono, biodiversidade e degradação evitada, como alternativa à arquitetura financeira}

\author{
Freddy G. Alvarado-Espinoza ${ }^{\mathrm{I}}$ \\ freddyalvaradorp@hotmail.com \\ Mauro C. Tapia-Toral II \\ mauro.tapiato@ug.edu.ec \\ Elaine R. Jijón-Gordillo III \\ elainejijon@hotmail.com
}

Correspondencia: freddyalvaradorp@ @otmail.com

\footnotetext{
I Magister en Negocios Internacionales y Gestión en Comercio Exterior, Diploma Superior en Gestión Empresarial Internacional, Diploma Superior en Autoevaluación con Fines de Acreditación, Economista, Docente de la Universidad Agraria del Ecuador, Docente de la Universidad de Guayaquil, Guayaquil, Ecuador.
}

II Magister en Finanzas y Proyectos Corporativos, Diploma Superior en Docencia Universitaria, Economista con Mención en Gestión Empresarial Especialización Finanzas, Contador Público Autorizado, Docente de la Universidad de Guayaquil, Guayaquil, Ecuador.

III Magister en Administración de Empresas, Magister en Administración de Negocios, Economista, Docente de la Universidad de Guayaquil, Guayaquil, Ecuador. 


\section{Resumen}

Este artículo, tuvo como objetivo analizar los certificados Yasuní dentro los postulados en el Protocolo de Kyoto, a fin de gestar ingresos fiscales y lograr una mejora de la calidad ambiental. La investigación fue de tipo descriptivo-explicativo-predictivo con un diseño no experimental. La población objeto de estudio estuvo constituida por el área del Parque Nacional Yasuní en el territorio del pueblo Waorani de la República del Ecuador, en una superficie total de 1,6 millones de hectáreas. El análisis de los resultados revela que, entre el mercado regulado y el mercado voluntario de carbono, a nivel mundial, entre los años 2006 y 2016 se comercializaron en promedio 8000 millones de toneladas de carbono. Para el Ecuador, una reserva de 846 millones de barriles dentro del parque equivale a 407 millones de $\mathrm{CO}^{2}$ que, al precio promedio de 20 USD la tonelada, significan un ingreso de alrededor de 8140 USD millones, una deforestación de 455 hectáreas de bosque y una movilización de 13589 turistas. Entre sus conclusiones, se determinó que el estudio de medir el impacto de los CGY sobre los ingresos fiscales y el medio ambiente, determina que las dos variables se ven afectadas en forma positiva cuando el precio del petróleo es de 32 y 52 dólares el barril, sin embargo, cuando el precio del petróleo es de $\$ 90$ el barril solo tenemos efectos ambientales positivos.

Palabras clave: ingresos fiscales; emisión de carbono; biodiversidad; degradación evitada.

\section{Abstract}

This article aimed to analyze the Yasuní certificates within the postulates in the Kyoto Protocol, in order to generate tax revenues and achieve an improvement in environmental quality. The research was descriptive-explanatory-predictive with a non-experimental design. The population under study was constituted by the area of the Yasuní National Park in the territory of the Waorani people of the Republic of Ecuador, in a total area of 1.6 million hectares. The analysis of the results reveals that, between the regulated market and the voluntary carbon market, worldwide, between the years 2006 and 2016 an average of 8,000 million tons of carbon were commercialized. For Ecuador, a reserve of 846 million barrels in the park equals 407 million $\mathrm{CO}^{2}$, which, at the average price of USD 20 per tonne, means an income of around USD 8140 million, a deforestation of 455 hectares of forest and a mobilization of 13589 tourists. Among its conclusions, it was determined that the study of measuring the impact of the CGY on fiscal revenues and the environment, determines that the two variables are affected in a positive way 
when the price of oil is 32 and 52 dollars a barrel, However, when the price of oil is $\$ 90$ a barrel, we only have positive environmental effects.

Keywords: tax revenues; carbon emission; biodiversity; degradation avoided.

\section{Resumo}

Este artigo teve como objetivo analisar os certificados Yasuní dentro dos postulados do Protocolo de Kyoto, a fim de gerar receitas fiscais e alcançar uma melhoria na qualidade ambiental. A pesquisa foi descritiva-explicativa-preditiva com delineamento não experimental. A população do estudo consistiu área do Parque Nacional Yasuni, no território das pessoas Waorani da República do Equador, em uma área total de 1,6 milhões de hectares. A análise dos resultados revela que, entre o mercado regulado e o mercado voluntário de carbono, mundialmente, entre os anos de 2006 e 2016, foram comercializadas, em média, 8.000 milhões de toneladas de carbono. Para o Equador, uma reserva de 846 milhões de barris nas quantidades parque para 407 milhões de $\mathrm{CO} 2$, o preço médio de 20 dólares por tonelada, significa uma renda de cerca de 8140 milhões de US \$, o desmatamento de 455 hectares de floresta e mobilização de 13589 turistas. Entre as suas conclusões, determinou-se que o estudo para medir o impacto de CGY sobre as receitas fiscais e ao meio ambiente, determina que as duas variáveis são afetados positivamente quando o preço do petróleo é de 32 e 52 dólares por barril, No entanto, quando o preço do petróleo é de US \$ 90 por barril, só temos efeitos ambientais positivos.

Palavras chave: receita tributária; emissão de carbono; biodiversidade; degradação evitada.

\section{Introducción}

Los problemas ambientales asociados a la contaminación antropógenas, dentro de las últimas décadas han incrementado su importancia, lo que ha desarrollado la conciencia ambiental respecto a los riesgos de la degradación de los ecosistemas, así como también los desgastes de los recursos naturales, particularmente los no renovables. Es así que la presión de los ambientalistas hacia los líderes de la comunidad internacional generan el inicio de acciones de tipo político que permiten afrontar la problemática ambiental; creándose limites a los niveles de contaminación como los generadores de gases de efecto invernadero, las mismas en el Convención de Marco realizada en las Naciones Unidas sobre el cambio climático, celebrada en mayo de 1992, se 
efectivizan; y se da inicio al convenio, reconociendo que los cambios climáticos y sus efectos adverso son de interés y preocupación para la comunidad.

El principal propósito de la convención es estabilizar las concentraciones de gases de efectos de invernadero (GEI) y desarrollar programas para la mitigación del cambio climático mediante la reducción de emisiones. Es así como en el contexto del marco de las Naciones Unidas sobre cambio climático, surge el llamado Protocolo de Kyoto en diciembre de 1997, el objetivo global de este protocolo es el compromiso de los países desarrollados y en vías al desarrollo para que, de manera conjunta, alcancen objetivos cuantificados de reducción de emisiones de GEI.

A partir de la vigencia del protocolo de Kyoto el mercado de carbono pasó de la teoría a la práctica. Se desarrolló el llamado mercado de carbono regulado y voluntario, dentro de este escenario global, el presente trabajo de investigación analiza la propuesta planteada por el Ecuador, denominada YASUNÍ - ITT, como una alternativa para contrarrestar el cambio climático.

\section{Desarrollo}

Para Petroproducción (2009), a través del mercado de carbono regulado y voluntario, se propone no extraer el petróleo de los campos Ishpingo, Tambococha y Tipituni (ITT) y por esa vía dejaría de enviarse al medio ambiente cerca de 407 millones de toneladas de CO2 en aproximadamente 13 años. En esta área petrolera, se estima que existen 846 millones de barriles en reservas, de petróleo con una vida útil de 25 años. Se proyecta que en los trece primeros años la producción ascienda a 107 mil barriles diarios, lo que en conjunto produciría ingresos para la economía ecuatoriana por alrededor de 14.300 millones de dólares, en caso de explotar el petróleo. (Petroproducción 2009).

\section{Mercado de carbono}

El mercado de carbono surge en el mundo como una vía complementaria, alternativa y económicamente viable al compromiso asumido por países, empresas e individuos, de disminuir las emisiones de gases que contribuyen al efecto invernadero (GEI). Este puede estar, según García (2011), dentro del cumplimiento y la observancia de las prerrogativas del Protocolo de Kyoto o puede estar en el mercado voluntario, el cual no es jurídicamente vinculante, pero se ha 
desarrollado como respuesta a aquellos que están interesados en convertirse en carbón neutral; es decir, se refiere a que diferentes instituciones implementan acciones de reducción de emisiones por diferentes motivos mediante instrumentos creados fuera de cualquier sistema regulado, los llamados no Kyoto.

\section{Biodiversidad}

La biodiversidad o diversidad biológica, para Barsev (2002), se refiere a la extensa variedad de seres vivos existentes en el planeta, es también un sistema dinámico que está en evolución constante. La distribución de la biodiversidad viene como consecuencia de factores evolutivos en el área biológica, geográfica y ecológica, por consiguiente, cada especie posee un entorno adecuado a sus necesidades en donde cada uno de estos puede relacionarse de manera armoniosa con el medio que lo rodea.

Entre los tipos de biodiversidad encontramos, la diversidad genética, la cual comprende la variación en los genes de una especie. Sucesivamente tenemos la diversidad de especie, la cual consiste en la cantidad de especies vivas que residen en un hábitat determinado. Luego está la diversidad ecológica, que es la variedad de ecosistemas o comunidades biológicas existentes en un área y el último tipo de biodiversidad es la diversidad funcional, es la variedad de respuesta de las especies a los cambios ambientales. Una de las principales amenazas de la biodiversidad ha sido el mismo ser humano, el cual por medio de sus acciones de deforestación, incendios y contaminación ha ocasionado daños que no solo afectan a las especies que habitan en esos lugares, sino que también han dañado al medio ambiente.

\section{Degradación ambiental}

La degradación ambiental, es todo proceso a través del cual el entorno natural se ve comprometido de alguna forma, se reduce la diversidad biológica o la salud general del medio se ve afectada. Los procesos de degradación ambiental pueden ser naturales en su origen o pueden ser causados por la actividad humana, que también puede acelerar procesos naturales de degradación. Muchas organizaciones internacionales, entre estas la Chicago Climate Exchange (2011), reconocen la degradación ambiental como uno de los principales ataques que está sufriendo el planeta, pues, por ahora, sólo tenemos un planeta en el que vivir y algunos daños irreparables podrían significar el final de la existencia humana. 


\section{Iniciativa Yasuní-ITT}

Según la Iniciativa Yasuní ITT el estado ecuatoriano se deberá comprometer a dejar bajo tierra, de forma indefinida, alrededor de 856 millones de barriles de petróleo en la reserva ecológica del Yasuní, para evitar la emisión a la atmósfera, de 407 millones de toneladas métricas de dióxido de carbono, que se producirían por la quema de esos combustibles fósiles, a cambio de una compensación económica de la comunidad internacional por una fracción del valor estimado por el 50\% de las utilidades que percibiría si se diera la explotación de este recurso (unos 350 millones de dólares anuales). Los fondos captados por esta operación se reinvertirían en el Ecuador en tres líneas: Manejo de 19 áreas protegidas, un programa de reforestación nacional y el cambio de matriz energética. Sobre los porcentajes dedicados a cada uno de estos fondos no hay consenso. Estos fondos serían manejados por un fideicomiso, sobre cuya integración, existen discrepancias entre los posibles aportantes y el gobierno ecuatoriano.

\section{Instrumentos financieros}

En el caso particular, de este artículo los instrumentos financieros utilizados en esta propuesta son: el mercado de carbono, donaciones y canje de la deuda y donaciones del sector privado.

Así mismo, los ingresos por la venta de certificados de garantía Yasuní según Cavalluci. (2009). provienen de las siguientes fuentes:

1) El CO2 evitado y medido en millones de toneladas y negociado en mercados voluntarios.

2) Compensaciones de biodiversidad.

3) Servicios hidrológicos.

4) Acuerdo NO OPEC por no explotar el petróleo.

5) Deforestación evitada.

6) Estrategia eficaz de Reducciones por Deforestación y Degradación (REDD). La propuesta de Ecuador es diferente a los postulados del Protocolo de Kyoto respecto a los mercados de carbono; en cuanto al efecto neto sobre el medio ambiente.

La diferencia principal entre el mecanismo de certificación Yasuní y el de derecho de emisión; es que el primero plantea evitar totalmente las emisiones, mientras que los certificados de reducción 
de emisiones persisten el problema ambiental. La propuesta apunta a crear un mecanismo para valorar estos certificados, además de servir de referencia para otras investigaciones similares.

\section{Materiales y métodos}

El tipo de estudio fue descriptivo, explicativo y predictivo orientado a conocer los atributos y desarrollo de la iniciativa Yasuní ITT, desde su génesis hasta la actualidad, relacionándolo con lo establecido en los acuerdos internacionales, en el contexto de la propuesta ecuatoriana. El diseño que se aplicó fue no experimental, dado que no existen grupos de control ni experimental. La población de la presente investigación estuvo constituida por el área de estudio del Parque Nacional Yasuní y el territorio del pueblo Waorani que juntos forman la reserva de la biosfera Yasuní. El método utilizado es el llamado de costos de oportunidad y se basa en la idea de que los costos de usar un recurso para objetivos que no tienen precios en el mercado o no son comercializados y se estiman usando el ingreso perdido por no usar el recurso.

Los datos del análisis lo constituyeron la oferta y demanda, en el caso de la oferta se determinaron los ingresos que genera la reserva, la misma que está relacionada con la biodiversidad, el costo de oportunidad de no explotar el petróleo, los servicios hidrológicos, las reducciones por deforestación y la degradación evitada. Por su parte, la demanda dependió de lo que la comunidad internacional esté dispuesta a contribuir para mantener la reserva ecológica, la que a su vez está influenciada por los precios de estos bonos y el mercado mundial.

Para determinar la incidencia de los certificados de garantía Yasuní en los ingresos fiscales y la contaminación ambiental, se utilizó el modelo de costos de oportunidad, considerando básicamente el recurso petrolero como ingreso que estaría sacrificando por dejar el petróleo en el subsuelo. Como técnica se utilizó la observación del sistema de valoración a través de precios de mercado y costo de oportunidad; y como estadística se aplicó un modelo de Regresión Múltiple.

\section{Análisis y discusión de los Resultados}

Los resultados del análisis de los datos del presente estudio, se realizaron a través de modelos matemáticos y estadísticos, presentación de resultados y análisis mediante el uso de tablas estadísticas inferencial de simple y doble entrada, así como ilustraciones que permiten observar la 
evolución de las variables en estudio (Los certificados Yasumi y la Arquitectura Financiera, 2013).

A partir de estos modelos, se pudo demostrar que los ingresos por emisiones reducidas dependen de: $\mathrm{CO} 2=\mathrm{Y}$ (toneladas) la misma que está en función de:

$\mathrm{X} 1=$ Compensación de biodiversidad.

X2 = Reforestación.

X3 = Costo de oportunidad de no explotar petróleo.

En el caso del costo de oportunidad es un método que se usa para valorar bienes que no tienen precios del mercado o no son comercializados y pueden ser estimados usando el ingreso perdido por no usar el recurso en otros usos alternativos.

Por el lado de los ingresos se han tomado en cuenta los beneficios ambientales, medido en términos de CO2 evitado, por no explotar el petróleo, el ecoturismo, los beneficios por biodiversidad y la reforestación. En el caso del petróleo se ha considerado de acuerdo a un diseño de producción de una empresa (Brasil-Francia), se calcula que solo en los 3 primeros años se explotaron 10 millones de barriles y que los 10 años restantes, se pueden explotar 40 millones de barriles, de allí que la producción llega a un punto crítico y declina.

Para determinar la cantidad de CO2 se usó el factor de conversión de 0,44 que resulta de dividir el total de reservas de petróleo y la cantidad de $\mathrm{CO} 2$ en millones de toneladas, es decir por cada barril de petróleo se genera 0,44 Ton. de CO2. Según Azqueta (2002), hay pérdida de \$100 por turista y al año llegan 20.000 turistas, en base a esto se estiman los ingresos y pérdidas.

En relación a la biodiversidad, según Azqueta (2002), se calculó el valor por hectárea, asumiendo como premisa que en los próximos 50 años habrá un impacto fuerte en la flora y fauna, en un 24\%, si se explota el petróleo. Al inicio la infraestructura que se levanta para explotar petróleo afecta el 10\% del área y en los próximos 10 años se afectará otro $10 \%$ y al final hasta los 50 años el 4\% restante, lo mismo para la reforestación, el gasto por reforestar es de $\$ 10.267$ por hectárea. Los 3 primeros años el 10\% y los otros 10 años es otro $10 \%$ y el resto $4 \%$ es del año 14 hasta el año 50 . 
La serie para el análisis es de 13 años, dado que es el tiempo que se estima que durarían las reservas de petróleo. Así mismo, se ha realizado un análisis de sensibilidad, considerando diversos escenarios, para el precio del petróleo, de 32, 50 y 90 dólares el barril, la tasa de descuento utilizada es del 5\%, considerada una tasa social y los precios para los bonos Yasuní están en \$20 dól/Ton, considerando los precios del mercado regulado y voluntario en el mercado mundial.

En los flujos de ingresos hasta el año 3, los ingresos generados por abatimiento $\mathrm{CO} 2$ se mantienen constantes, mientras que a partir de este año aumentan debido a que cambió el volumen de extracción del crudo. Así mismo, se ha utilizado el valor presente neto y la relación beneficio costo.

Los resultados son los siguientes. A un precio del petróleo de 32 dólares el barril el VPN de los ingresos es sustancialmente el triple al VPN de los egresos y además se mantiene una relación beneficio-costo de 3,6.

Por su parte, a medida que aumenta el precio del petróleo, el costo de oportunidad de no explotar este recurso aumenta, con lo cual la relación beneficio-costo disminuye a 1.9 y el VPN ingresos es el doble al VPN de los gastos.

En el tercer caso se ha estimado un precio del petróleo de 90 dólares, con lo cual el valor presente neto de los egresos ligeramente mayor al VPN de los ingresos, manteniendo una relación beneficiocosto menor a 1, lo que de acuerdo a los resultados se estaría justificando la no venta de los certificados de garantía Yasuní. Sin embargo, tampoco es justificación para explotar las reservas, a pesar de que el Ecuador es un país que requiere de los recursos petroleros.

Con este modelo se validó la hipótesis que estos certificados de garantía generan ingresos fiscales y al mismo tiempo mejoran la calidad ambiental. En las tablas $\mathrm{N}^{\circ} 1,2$ y 3 se puede ver el impacto de los certificados Yasuní en los ingresos fiscales, así como las proyecciones en tres diferentes escenarios económicos del precio del barril del petróleo para los 4 primeros años de explotación y su proyección al año 13ro.

En la cuantificación del impacto de los certificados Yasuní en los ingresos fiscales y la emisión de carbono, la biodiversidad y la degradación evitada se tomó en cuenta el tiempo de la 
explotación de la reserva de petróleo, 13 años. Económicamente se realizó el análisis de sensibilidad teniendo presente el precio del barril de petróleo en 32.50 y 90 dólares, un 5\% de tasa de descuento que el precio por tonelada, de CO2 de \$20 dólares, según los análisis econométricos se ha podido ver que el VPN de los ingresos por ejemplo para 32 dólares el barril de petróleo que se dejaría de explotar, es el triple del VPN de los egresos, y con una relación de B/C de 3.6, valor etc. Que lo consideramos muy atractivo para los inversionistas privados, y es notorio la disminución de la relación B/C a medida que aumenta el barril del petróleo, llegando inclusive a números de 1 cuanto éste costo del barril se sensibiliza en el modelo con 90 dólares. 
Freddy G. Alvarado Espinoza, Mauro C. Tapia Toral, Elaine R. Jijón Gordillo

Tabla 1. Impactos de los Certificados Yasuní en los Ingresos Fiscales

\begin{tabular}{|c|c|c|c|c|c|c|c|c|c|c|c|}
\hline \multirow{2}{*}{ CONCEPTO } & \multirow{2}{*}{$\begin{array}{l}\text { COSTO } \\
\text { UNITARIO }\end{array}$} & \multicolumn{2}{|c|}{ 1er AÑo } & \multicolumn{2}{|c|}{ 2do AÑo } & \multicolumn{2}{|r|}{ 3er AÑo } & \multicolumn{2}{|r|}{ 4to AÑo } & \multicolumn{2}{|r|}{ 5to AÑo } \\
\hline & & UNIDAD & VALOR & UNIDAD & VALOR & UNIDAD & VALOR & UNIDAD & VALOR & UNIDAD & VALOR \\
\hline \multicolumn{12}{|l|}{$\begin{array}{l}\text { EGRESOS (COSTO DE } \\
\text { OPORTUNIDAD) }\end{array}$} \\
\hline $\begin{array}{l}\text { Producción Petrolera del } \\
\text { - Bloque ITT }\end{array}$ & 20,00 & 10000000 & $200.000 .000,00$ & 10000000 & $200.000 .000,00$ & 10000000 & $200.000 .000,00$ & 40000000 & $800.000 .000,00$ & 40000000 & $800.000 .000,00$ \\
\hline TOTAL EGRESOS & & & $200.000 .000,00$ & & $200.000 .000,00$ & & $200.000 .000,00$ & & $800.000 .000,00$ & & $800.000 .000,00$ \\
\hline \multicolumn{12}{|l|}{$\begin{array}{l}\text { INGRESOS } \\
\text { (BENEFICIOS } \\
\text { AMBIENTALES) } \\
\end{array}$} \\
\hline - Abatamiento del $\mathrm{CO} 2$ & 20,00 & 4400000 & $88.000 .000,00$ & 4400000 & $88.000 .000,00$ & 4400000 & $88.000 .000,00$ & 17600000 & $352.000 .000,00$ & 17600000 & $352.000 .000,00$ \\
\hline - Eco turismo & 100,00 & 20000 & $2.000 .000,00$ & 20000 & $2.000 .000,00$ & 20000 & $2.000 .000,00$ & 20000 & $2.000 .000,00$ & 20000 & $2.000 .000,00$ \\
\hline - Biodiversidad & $1.600,00$ & 98200 & $157.120 .000,00$ & 98200 & $157.120 .000,00$ & 98200 & $157.120 .000,00$ & 196400 & $314.240 .000,00$ & 196400 & $314.240 .000,00$ \\
\hline - Reforestación & $10.267,00$ & 98200 & $1.008 .219 .400,00$ & 98200 & $1.008 .219 .400,00$ & 98200 & $1.008 .219 .400,00$ & 196400 & $2.016 .438 .800,00$ & 196400 & $2.016 .438 .800,00$ \\
\hline TOTAL INGRESOS & & & $1.255 .339 .400,00$ & & $1.255 .339 .400,00$ & & $1.255 .339 .400,00$ & & $2.684 .678 .800,00$ & & $2.684 .678 .800,00$ \\
\hline UTILIDAD & & & $1.055 .339 .400,00$ & & $1.055 .339 .400,00$ & & $1.055 .339 .400,00$ & & $1.884 .678 .800,00$ & & $1.884 .678 .800,00$ \\
\hline
\end{tabular}

\begin{tabular}{|c|c|c|c|c|c|c|c|c|c|c|c|}
\hline \multirow{2}{*}{ CONCEPTO } & \multirow{2}{*}{$\begin{array}{l}\text { COSTO } \\
\text { UNITARIO }\end{array}$} & \multicolumn{2}{|r|}{ 6to AÑo } & \multicolumn{2}{|r|}{$7 \mathrm{mo}$ AÑo } & \multicolumn{2}{|r|}{ 8vo AÑo } & \multicolumn{2}{|r|}{ 9no AÑo } & \multicolumn{2}{|r|}{$10 \mathrm{mo}$ AÑo } \\
\hline & & UNIDAD & VALOR & UNIDAD & VALOR & UNIDAD & VALOR & UNIDAD & VALOR & UNIDAD & VALOR \\
\hline \multicolumn{12}{|l|}{$\begin{array}{l}\text { EGRESOS (COSTO DE } \\
\text { OPORTUNIDAD) } \\
\end{array}$} \\
\hline $\begin{array}{l}\text { Producción Petrolera del } \\
\text { - Bloque ITT }\end{array}$ & 20,00 & 40000000 & $800.000 .000,00$ & 40000000 & $800.000 .000,00$ & 40000000 & $800.000 .000,00$ & 40000000 & $800.000 .000,00$ & 40000000 & $800.000 .000,00$ \\
\hline TOTAL EGRESOS & & \multicolumn{2}{|r|}{$800.000 .000,00$} & \multicolumn{2}{|r|}{$800.000 .000,00$} & \multicolumn{3}{|c|}{$800.000 .000,00$} & $800.000 .000,00$ & \multicolumn{2}{|r|}{$800.000 .000,00$} \\
\hline \multicolumn{12}{|l|}{$\begin{array}{l}\text { INGRESOS } \\
\text { (BENEFICIOS } \\
\text { AMBIENTALES) } \\
\end{array}$} \\
\hline - Abatamiento del $\mathrm{CO} 2$ & 20,00 & 17600000 & $352.000 .000,00$ & 17600000 & $352.000 .000,00$ & 17600000 & $352.000 .000,00$ & 17600000 & $352.000 .000,00$ & 17600000 & $352.000 .000,00$ \\
\hline - Eco turismo & 100,00 & 20000 & $2.000 .000,00$ & 20000 & $2.000 .000,00$ & 20000 & $2.000 .000,00$ & 20000 & $2.000 .000,00$ & 20000 & $2.000 .000,00$ \\
\hline - Biodiversidad & $1.600,00$ & 196400 & $314.240 .000,00$ & 196400 & $314.240 .000,00$ & 196400 & $314.240 .000,00$ & 196400 & $314.240 .000,00$ & 196400 & $314.240 .000,00$ \\
\hline - Reforestación & $10.267,00$ & 196400 & $2.016 .438 .800,00$ & 196400 & $2.016 .438 .800,00$ & 196400 & $2.016 .438 .800,00$ & 196400 & $2.016 .438 .800,00$ & 196400 & $2.016 .438 .800,00$ \\
\hline TOTAL INGRESOS & & & $2.684 .678 .800,00$ & & $2.684 .678 .800,00$ & & $2.684 .678 .800,00$ & & $2.684 .678 .800,00$ & & $2.684 .678 .800,00$ \\
\hline UTILIDAD & & & $1.884 .678 .800,00$ & & $1.884 .678 .800,00$ & & $1.884 .678 .800,00$ & & $1.884 .678 .800,00$ & & $1.884 .678 .800,00$ \\
\hline
\end{tabular}

Pol. Con. (Edición núm. 21) Vol. 3, No 7, julio 2018, pp. 331-346, ISSN: 2550 - 682X 
Los ingresos fiscales en la emisión de carbono, biodiversidad y la degradación evitada, como alternativa a la arquitectura financiera

\begin{tabular}{|c|c|c|c|c|c|c|c|c|c|}
\hline \multirow{2}{*}{ CONCEPTO } & \multirow{2}{*}{$\begin{array}{l}\text { COSTO } \\
\text { UNITARIO }\end{array}$} & \multicolumn{2}{|r|}{$10 \mathrm{mo}$ AÑo } & \multicolumn{2}{|r|}{ 11ro AÑo } & \multicolumn{2}{|r|}{ 12do AÑo } & \multicolumn{2}{|r|}{ 13ro AÑo } \\
\hline & & UNIDAD & VALOR & UNIDAD & VALOR & UNIDAD & VALOR & UNIDAD & VALOR \\
\hline \multicolumn{10}{|c|}{ EGRESOS (COSTO DE OPORTUNIDAD) } \\
\hline $\begin{array}{l}\text { Producción Petrolera del Bloque } \\
\text { - ITT }\end{array}$ & 20,00 & 40000000 & $800.000 .000,00$ & 40000000 & $800.000 .000,00$ & 40000000 & $800.000 .000,00$ & 40000000 & $800.000 .000,00$ \\
\hline TOTAL EGRESOS & & & $800.000 .000,00$ & & $800.000 .000,00$ & & $800.000 .000,00$ & & $800.000 .000,00$ \\
\hline \multicolumn{10}{|l|}{$\begin{array}{l}\text { INGRESOS (BENEFICIOS } \\
\text { AMBIENTALES) } \\
\end{array}$} \\
\hline - Abatamiento del $\mathrm{CO} 2$ & 20,00 & 17600000 & $352.000 .000,00$ & 17600000 & $352.000 .000,00$ & 17600000 & $352.000 .000,00$ & 17600000 & $352.000 .000,00$ \\
\hline - Eco turismo & 100,00 & 20000 & $2.000 .000,00$ & 20000 & $2.000 .000,00$ & 20000 & $2.000 .000,00$ & 20000 & $2.000 .000,00$ \\
\hline - Biodiversidad & $1.600,00$ & 196400 & $314.240 .000,00$ & 196400 & $314.240 .000,00$ & 196400 & $314.240 .000,00$ & 196400 & $314.240 .000,00$ \\
\hline - Reforestación & $10.267,00$ & 196400 & $2.016 .438 .800,00$ & 196400 & $2.016 .438 .800,00$ & 196400 & $2.016 .438 .800,00$ & 196400 & $2.016 .438 .800,00$ \\
\hline TOTAL INGRESOS & & & $2.684 .678 .800,00$ & & $2.684 .678 .800,00$ & & $2.684 .678 .800,00$ & & $2.684 .678 .800,00$ \\
\hline
\end{tabular}

Fuente y elaboración: Autores 
Tabla 2. Tasa Social de Descuento

Precio 32 dólares

dólares
Precio 50 dólares

Precio 90

\begin{tabular}{|c|c|c|c|c|c|c|c|}
\hline \multirow{4}{*}{$\begin{array}{l}\text { Tasa } \\
\text { social de } \\
\text { descuento }\end{array}$} & \multirow[t]{4}{*}{$5,00 \%$} & VPN & $\$$ & VPN & $\$ 21.326 .280 .550,35$ & VPN & $\$$ \\
\hline & & & & & \multicolumn{3}{|c|}{ (Ingresos) } \\
\hline & & $\begin{array}{c}\text { VPN } \\
\text { (Egresos) }\end{array}$ & $\$ 5.880 .909 .572,05$ & $\begin{array}{c}\text { VPN } \\
\text { (Egresos) }\end{array}$ & $\$ 11.173 .728 .186,90$ & $\begin{array}{c}\text { VPN } \\
\text { (Egresos) }\end{array}$ & $\begin{array}{r}\$ \\
22.935 .547 .331,01\end{array}$ \\
\hline & & $\mathrm{RB} / \mathrm{C}$ & 3,626357503 & $\mathrm{RB} / \mathrm{C}$ & 1,908609212 & $\mathrm{RB} / \mathrm{C}$ & 0,929835257 \\
\hline
\end{tabular}

Fuente y elaboración: Autores 
Tabla 3. Análisis de Sensibilidad

\section{PRECIO DE 32 DÓLARES}

\begin{tabular}{|c|c|c|c|c|c|c|c|c|c|}
\hline $\begin{array}{l}\text { EGRESOS (COSTO DE } \\
\text { OPORTUNIDAD) }\end{array}$ & & & 1er AÑO & & 2do AÑO & & 3er AÑO & & 4to AÑO \\
\hline $\begin{array}{l}\text { Producción Petrolera del Bloque } \\
\text { - ITT }\end{array}$ & 20,00 & 10000000 & $200.000 .000,00$ & 10000000 & $200.000 .000,00$ & 10000000 & $200.000 .000,00$ & 40000000 & $800.000 .000,00$ \\
\hline TOTAL EGRESOS & & & $200.000 .000,00$ & & $200.000 .000,00$ & & $200.000 .000,00$ & & $800.000 .000,00$ \\
\hline
\end{tabular}

Nota: Desde el 4to año hasta el 13ero Año los valores de producción petrolera del Bloque ITT y el total de egresos son iguales.

\section{PRECIO DE 50 DÓLARES}

\begin{tabular}{|c|c|c|c|c|c|c|c|c|c|}
\hline \multicolumn{3}{|c|}{ EGRESOS (COSTO DE OPORTUNIDAD) } & \multicolumn{2}{|l|}{ 1er AÑ̃ } & \multicolumn{2}{|l|}{ 2do AÑO } & \multicolumn{2}{|l|}{ 3er AÑO } & 4to AÑO \\
\hline $\begin{array}{l}\text { - } \quad \text { Producción Petrolera del Bloque } \\
\text { ITT. }\end{array}$ & 38,00 & 10000000 & $380.000 .000,00$ & 10000000 & $380.000 .000,00$ & 10000000 & $380.000 .000,00$ & 40000000 & $1.520 .000 .000,00$ \\
\hline TOTAL EGRESOS & & & $380.000 .000,00$ & & $380.000 .000,00$ & & $380.000 .000,00$ & & $1.520 .000 .000,00$ \\
\hline
\end{tabular}

Nota: Desde el 4to año hasta el 13ero Año los valores de producción petrolera del Bloque ITT y el total de egresos son iguales.

\section{PRECIO DE 90 DÓLARES}

\begin{tabular}{|c|c|c|c|c|c|c|c|c|c|}
\hline $\begin{array}{l}\text { EGRESOS (COSTO DE } \\
\text { OPORTUNIDAD) }\end{array}$ & & & 1er AÑO & & 2do AÑO & & 3er AÑO & & 4to AÑO \\
\hline $\begin{array}{l}\text { Producción Petrolera del } \\
\text { - } \quad \text { Bloque ITT }\end{array}$ & 78,00 & 10000000 & $780.000 .000,00$ & 10000000 & $780.000 .000,00$ & 10000000 & $780.000 .000,00$ & 40000000 & $3.120 .000 .000,00$ \\
\hline TOTAL EGRESOS & & & $780.000 .000,00$ & & $780.000 .000,00$ & & $780.000 .000,00$ & & $3.120 .000 .000,00$ \\
\hline
\end{tabular}

Nota: Desde el 4to año hasta el 13ero Año los valores de producción petrolera del Bloque ITT y el total de egresos son iguales. Fuente y elaboración: Autor 


\section{Conclusiones}

Valorizar el medio ambiente y los recursos naturales comunes no es una tarea fácil, dado que en muchos de los casos no asisten al mercado, por lo tanto, requieren de otros mecanismos como: el método de valoración directo, que usa técnicas basadas en experimentos y encuestas. El indirecto a través de encuestas, donde mide las preferencias de los consumidores. También tenemos métodos de valoración de beneficios y costos.

En cuanto a los certificados de garantía Yasuní, existen diferencias concretas entre los bonos de carbono y estos certificados, esto es la CGY, son certificados de no emisión o emisiones evitadas, mientras que los primeros son productos medidos en CO2 que se comercializan, además los CGY pueden desaparecer si no se cumple con la normativa de no deforestación evitada, el ecoturismo, la biodiversidad y la conservación de los llamados grupos sociales en aislamiento voluntario. En relación a los costos determinados los ingresos perdidos por no explotar petróleo, afecta a los ingresos fiscales, los gastos públicos, el PIB y las exportaciones.

El estudio de medir el impacto de los CGY sobre los ingresos fiscales y el medio ambiente determina que las dos variables se ven afectadas en forma positiva cuando el precio del petróleo es de 32 y 52 dólares el barril, sin embargo, cuando el precio del petróleo es de $\$ 90$ el barril solo tenemos efectos ambientales positivos.

\section{Referencias Bibliográficas}

Azqueta, D. (2002). Introducción a la Economía Ambiental. España: Mac Graw Hill.

Banco Mundial. (2005). Community Development Carbon Fund Annual Report 2004.

Washitong: Word Bank.

Barsev (2002) Metodología de valoración de bienes ambientales

Cavalluci, O. (2009). El esquema Europeo de compra - venta de Derechos de Emisión (EU-ETS) y la iniciativa Yasuní ITT. Quito: Consejo de Administrativo y Directivo Iniciativa Yasuní-ITT.

Chicago Climate Exchange. (30 de Junio de 2011). ICE Global markets in clear view.

Recuperado el 18 de 02 de 2012, de Chicago Climate Exchange:

https://www.theice.com/publicdocs/ccx/CCX_Fact_Sheet.pdf 
García, D (2011) Mercado del carbono y América latina (Parte I). Perú: Bioalejandría. http://bioalejandria.blogspot.com/2011/05/el-mercado-del-carbono-parte-i.html

[Acceso: 24/09/2011]

Iniciativa Yasuni

ITT. (2007).

ITT\&aqs=chrome.3.69i57j69i60j014.12125j0j7\&sourceid=chrome\&ie=UTF-8rio/informes/807informe-de-la-inspeccion-en-el-parque-nacional-yasuni.Recuperado en julio 2017.

Los certificados Yasuni y la Arquitectura Financiera (2013). http://www.accionecologica.org/bosques-y-plantaciones/aislamiento-volunta https://www.google.com.ec/search?q=iniciativa+yasuni+itt+objetivos\&oq=Iniciativa+Yasun\%C3 $\%$ AD- Recuperado en junio 2016.

Petroecuador (2009). https://www.bnamericas.com/company-profile/es/empresa-publica-dehidrocarburos-del-ecuador-ep-petroecuador. Recuperado el 13 de mayo 2017 